\title{
QoS-Based Two-Level user Scheduling Scheme for MU-MIMO Wireless LANs
}

\author{
D. Srinivasa Rao, V. Berlin Hency
}

\begin{abstract}
The advances in physical layer technology has led to the performance upgradation of wireless local area networks (WLANs). More recently, multi-input multi-output (MIMO) is considered to be a key technology to enable high data rate transmission in WLANs. However, the actual benefit of this approach can be utilized, if there is an appropriate mechanism to select and schedule the users. Also, providing Quality of Service (QoS) support to user demands has become a major task in WLANs. In this paper, a two-level user scheduling approach for WLANs is discussed and its performance is evaluated using high-transmission rates with the assumption of frequency selective fading. For the purpose of comparison, some well-known medium access control (MAC) scheduling schemes are considered. It is shown that, the proposed scheme enhances throughput and achieves fairness among the users. Further, this scheme can be used to reduce contention during the acquisition of channel feedback.
\end{abstract}

Keywords: quality of service, channel feedback, scheduler, throughput, fairness

\section{INTRODUCTION}

$\mathrm{T}$ he standardization of IEEE 802.11 based WLAN and its wide deployment has resulted in increased usage of the internet in many places. Channel aggregation, higher order modulation schemes, and MIMO are some of the crucial physical layer techniques adopted in current WLANs [1]. With the introduction of multiple antenna techniques [2], the raw rates offered by these networks have reached gigabit speeds [3]. This allows users to demand for high-bandwidth applications like online video streaming and transfer of multimedia data. At the same time, the number of consumers availing the services of WLANs has also increased drastically in the past decade. However, the actual rate experienced by the users may be affected if the resources are not utilized properly. Hence, a notable gain in the performance can be attained, if an efficient resource management mechanism is adopted above the physical layer. Conventionally, resource allocation and scheduling are considered as key approaches to handle these issues. Acquisition of channel state information (CSI) is the fundamental step in selecting users and allocating resources to them. But the problem with multi-user scenario is, CSI from all the users should be obtained to schedule the users and offer the required QoS. This results in inefficient usage of bandwidth and ultimately lead to degradation of throughput performance. So, to enhance the overall throughput performance, it is vital to devise suitable scheduling mechanism for these networks.

D. Srinivasa Rao, Department of ECE, GMR Institute of technology, Rajam, AP, India. Email: srinivasarao.d@gmrit.edu.in

School of Electronics Engineering, VIT, Chennai, Tamil Nadu, India

V. Berlin Hency, School of Electronics Engineering, VIT, Chennai, Tamil Nadu, India. Email: berlinhency.victor@vit.ac.in
Revised Manuscript Received on December 02, 2019

There are several contributions in literature, that have focused on throughput performance by considering the key features offered in physical and medium access layer of 802.11 WLANs. Some of them are discussed in this section. Performance analysis is the fundamental task that is to be carried to study the impact of various features that were developed for any system. For instance, the author in [4], has evaluated the performance of $802.11 \mathrm{ac}$ by using channel adaptation and block precoding approach with varied MIMO detection methods. Similarly, in [5], the author presented a simulation framework to analyse and improve the physical layer attributes of $802.11 \mathrm{ac}$ under multi-user scenarios. Another study in [6], has completely analysed the system throughput performance of $802.11 \mathrm{ac}$ by considering features like modulation rates, number of access point antennas and channel bandwidth. Another notable attribute that enhances throughput in 802.11 WLANs is frame aggregation. By combining and transmitting multiple packets together reduces the overhead in the entire process. In [7], the author has shown that, with the help of packet aggregation and by using spatial multiplexing, the throughput performance can be significantly improved. The authors in [8], evaluated the performance of downlink multi-user WLAN by using frame aggregation and space time block codes. It was shown that frame aggregation with spatial multiplexing achieves better performance in the case of throughput in contrast to the single user transmission scheme. The authors in [9], proposed an efficient frame aggregation approach that advances throughput and reduce the frame error rate in downlink multi-user MIMO WLANs. Channel state acquisition in multi-user MIMO WLANs has gained attention, because it can extend the transmission capability of the system by reducing the overhead requirements.

In [10], the author has shown that with appropriate implicit training mechanism, the capability of downlink Multi user-MIMO WLAN can be extended and significant performance gain is achieved over beam-forming approach. Adaptive compression of channel state information is implemented in [11] to reduce the feedback overhead due to multiple requests. It is a simple mechanism that adapts compression intensity to optimize network throughput. The author in [12], has compared the implicit and explicit feedback mechanisms in WLANs with respect to overhead specification and packet error rate. In order to share the resources among the contending users, some resource controlling strategy is always needed in any wireless system. In particular, the design of an efficient MAC has got a lot of significance, as it should be adapted to the dynamically changing physical aspects of multi-user MIMO WLANs. 


\section{QoS-Based Two-Level user Scheduling Scheme for MU-MIMO Wireless LANs}

The basic MAC enhancements for high throughput wireless LANs were briefly discussed in [13], with focus on transmit opportunity mechanism (TXOP). The author also evaluated the proposed mechanism using system level simulations. A rational markov chain model is proposed in [14], to measure the IEEE 802.11ac performance by allowing TXOP sharing approach. The ultimate goal of this work is to analyse how this approach might enhance the usage of the sparse wireless bandwidth while attaining channel access fairness within the various access categories (ACs). With the aim of throughput maximization in IEEE 802.11ac, the author in [15] proposed a throughput-aware TXOP sharing scheme that implements joint link adaptation and scheduling of the downlink multiuser transmission. By using this approach, significant gain in MAC layer throughput is obtained. However, in most of the works discussed above, the task of resource allocation and scheduling is considered separately. Therefore, to explore the full potential of multi-user MIMO WLANs, the physical and medium access parameters are to be jointly considered. Hence, in this paper, we propose a two-level user scheduling scheme (TLUS) that considers physical aspects such as CSI feedback, modulation order and spatial multiplexing along with QoS requirements. Access point (AP) performs the scheduling in two levels. In the first level, the access point prepares the list of active users through a contention based feedback mechanism. In the next level, user grouping and scheduling will be performed based on individual transmission time intervals. The major objectives of the proposed scheme are as follows:

- It achieves fairness among the users by selecting the best users and allocating the resources based on QoS requirements.

- Also, throughput performance can be improved by scheduling the users according to relative transmission durations.

To yield better results, the combined effect of modulation, spatial streams and scheduling is studied using simulation. From the results, it is shown that resource scheduling with higher modulation and increased spatial streams improves overall throughput performance of the network system.

The remaining content of the paper is structured in the following order. In section II, the related work involving the problem is highlighted. In section III, the description of the proposed TLUS design is given and the key parameters chosen to study the impact on throughput performance is also discussed. In section IV, the evaluation of the proposed scheme is discussed with the help of simulation results. Lastly, section $\mathrm{V}$ gives the concluding remarks of the paper.

\section{RELATED WORK}

The provision of QoS in WLANs is not a recent subject matter; it was introduced in earlier amendment $802.11 \mathrm{e}$ to offer QoS services [16] to multimedia users. Controlled and prioritized channel access to the users improves the quality of service. This is usually referred to as scheduling. That is, QoS relies majorly on allocation of resources and proper scheduling. However, with the increase in demand for multimedia applications, the number of users availing the network services has also increased significantly. The introduction of MIMO technology in WLANs has boosted the offered raw rates at the physical layer. Now, the major challenge is to upgrade the medium access layer of 802.11 to accommodate multiple users. This is considered as the motivation behind our work, and here we discuss about some of the recent works relating to QoS scheduling in WLANs. The task of scheduling involves user selection, grouping and scheduled transmission. Wireless networks are highly vulnerable to noise and interference. The channel states of users contending for sources may not be same all the time. They vary rapidly due to the disturbances present inside and outside the network. Selecting the users with good channel conditions is essential in resource allocation. This will reduce the wastage of bandwidth and improves the throughput of the system. Proper user selection will also reduce the interference among the users contending for resources. Obtaining CSI from the users in the network is the first step in the process of user selection. It can be done either implicitly or explicitly [10]. In the implicit feedback mechanism, the AP quantifies the CSI by estimating the training sequences sent by user stations (STA) and in explicit feedback, the user stations will send the channel states to AP. The major problem with CSI feedback is increased overhead, channel sounding interval and frequency of CSI requests. CSI feedback compression and overhead reduction is discussed in [11] [12]. The impact of channel sounding interval is studied in [17] [18]. Once, the channel information is obtained, the AP scheduler selects the users to form an optimal user set which can maximize the overall throughput. However, this becomes a complex problem when the user size is sufficiently large. Hence, this necessitates the need for an efficient user selection mechanism. The current 802.11ac standard employs random user selection (RUS) [19] mechanism where all the users are selected randomly with equal probability. But, this approach incurs large overhead when the number of users increases. The author in [20], has proposed a low complexity semi-orthogonal user selection mechanism that selects optimal user set based on highest channel qualities. Zero-forcing beamforming is used to mitigate the interference between the users. In another work [21], the author has evaluated the orthogonality among various users and proposed a distributed contention mechanism to eliminate the need to obtain CSI from each and every user in the network. The users are required to send feedback only, when they met the orthogonality conditions. This is suitable for networks with large set of users. The author in [22], jointly considered sounding, selection and scheduling of users to improve throughput and provide fairness. In [23], the author suggested a novel orthogonality based contention process known as signpost to select users in uplink direction. Another uplink user selection approach referred to as optimal user selection mechanism is presented in [24] that provides throughput and fairness solution to complex scheduling problem. The author in [18], decoupled the user sounding and selection process that reduces the overhead incurred in channel feedback process. In [25], the author has proposed a channel pre-sounding mechanism to select the appropriate mode and users prior to sounding process. It was shown that the method achieves maximum throughput compared to other fixed mode policies. 
User scheduling based on QoS requirements has gained lot of prominence after the introduction of MIMO feature in WLANs. Selecting optimal user set and schedule them to obtain maximum throughput and fairness has become the objective of current WLANs. Here, we review some of the scheduling mechanisms that have improved the utilization of MU-MIMO capability in WLANs. In [26], the author considered the issue of fairness in MU-MIMO WLANs and proposed a multi-user scheduling scheme. The scheme achieves high degree of fairness by simultaneous transmissions to various users. The author in [27], proposed a low-complexity MU-MIMO scheduling scheme using block diagonalization with chordal distance. It was shown that, by using this method, optimal throughput can be achieved. The author in [28], considered a distributed opportunistic scheduling scheme and users can able to contend for resources if they exceed the threshold transmission rate. The throughput and delay performance is analysed under the influence of physical and MAC parameters. In another work [29], the author proposed a SNR threshold based distributed multiuser scheduling scheme to enhance the throughput performance of WLANs. Here, the author considered link quality, collision probability and protocol overhead to optimize the throughput performance of the system. To further extend the utilization capability MU-MIMO, the author in [22], has proposed an active CSI feedback based user scheduling scheme to elevate the throughput gain and provide better fairness among the users. Recently, in [30], the author proposed a joint user selection and scheduling (JUSS) scheme that uses a dual alignment metric to pick out the desired user set and schedule them concurrently. The proposed scheme enhances throughput, reduces contention, eliminates interference and provides fairness among the users. In all the works that were reviewed in this section, the major aspects addressed are throughput, complexity and fairness. Transmission time and scheduling duration are crucial resources in WLANs. Here, we introduce a two level user scheduling scheme that considers CSI feedback along with time constraints from upper layer to efficiently schedule the users. The brief narration of the proposed scheme is given in the next section.

\section{TLUS DESIGN}

In this part, we discuss about the system model, design overview and then present the two-level scheduling algorithm. We also discuss about the constraints that are chosen to enhance the throughput performance of WLANs.

\section{A. System model}

The downlink of a Wi-Fi infrastructure network comprises of ' $\mathrm{K}$ ' user stations with one or more antennas, and an access point which consists of ' $M$ ' number of antennas. The entire WLAN scenario is depicted in Fig. 1. The assumption is that the AP contains adequate data to transmit for the user download purpose. The serving order and amount of data the users receive will be in accordance with the channel circumstances and QoS demands. The data will be transmitted through the AP to user STAs using multiple antennas. The channel varies in accordance with time and satisfies rayleigh fading conditions. Let $\mathrm{k}=1,2,3, \ldots \ldots, \mathrm{N}$ be the number of users and $\mathrm{x}_{\mathrm{k}}$ be the transmitted symbols. $\mathrm{H}_{\mathrm{k}}$ be the channel matrix between transmit antennas and receivers, $\mathrm{n}_{\mathrm{k}}$ is the noise vector of kth user. Then, the received signal of the MU-MIMO system is given by,

$$
y_{k}=H_{k} x_{k}+n_{k}
$$

Though, Zero-force beamforming (ZFBF) is the simplest method, here minimum mean square error (MMSE) precoding is used as it suppresses the noise and eliminates the interference.

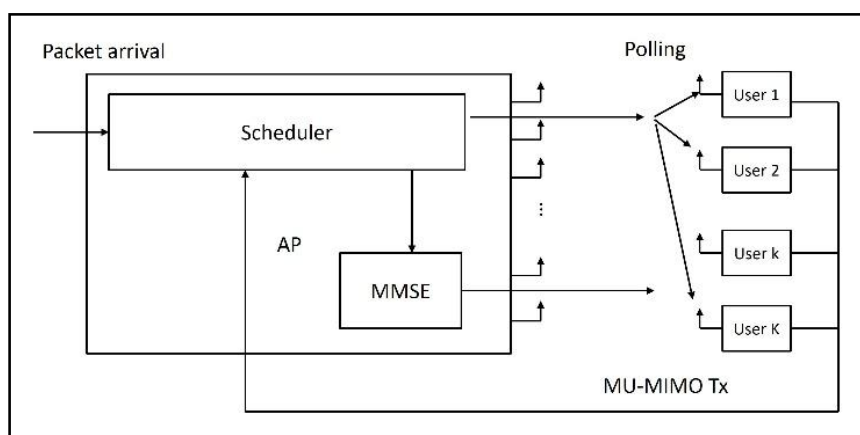

Fig. 1. System model

The principle of orthogonality among the multiple data streams leads to uncorrelated symbols at any instant. Let $\mathrm{w}_{\mathrm{k}}$ be the beamforming vector and $\mathrm{S}$ be the selected user group, and then the optimal precoding vector for interference cancellation is given by,

$$
W(S)=P_{t}\left[P_{t} H(S) * H(S)+\sigma_{n}^{2} I\right]^{-1} H(S)^{*}
$$

Here, Pt corresponds to the power of transmitted data symbols, $\sigma_{n}^{2}$ represents the noise vector and $\mathrm{H}$ be the channel matrix. $\mathrm{H}^{*}$ be the conjugate transpose matrix.

\section{B. Design overview}

In the legacy WLANs like $802.11 \mathrm{a} / \mathrm{b} / \mathrm{g}$, the basic medium access technique is distributed coordination function (DCF). It is based upon carrier sense multiple access/collision avoidance (CSMA/CA) protocol. These protocols are typically meant for serving single user stations. However, with the introduction of MIMO in 802.11n, APs are equipped with multiple antennas to serve many users at the same time. The multi-user transmission feature adopted in these networks is known as enhanced distributed channel access (EDCA). The TXOP sharing mechanism is the first enhancement in the basic MAC function of current 802.11 WLANs. It aims at achieving better QoS and has been implemented as the fundamental sharing mechanism in all high throughput wireless local area networks. In the IEEE 802.11ac standard, the downlink multi-user MIMO transmission is planned according to the TXOP sharing rules. The AP will first contend for medium to attain a TXOP and attempts to reserve radio resources according to the traditional DCF on behalf of all the stations. After obtaining the resources, the AP collects the CSI of each station, and allocates subsets of these radio resources essentially OFDM subcarriers and time slots to different stations for simultaneous transmission during the TXOP. 


\section{QoS-Based Two-Level user Scheduling Scheme for MU-MIMO Wireless LANs}

In the downlink scenario, AP is responsible for preparation of multi-user transmission schedule. This differentiates the scheduler design among various MAC layer protocols. Our design selects the users based on effective channel gains and schedules them according to the transmit time durations.

Generally, the transmission rates of the users will depend on the type of application, queue size in the buffer and channel conditions. Considering these factors for scheduling decision is a preferable way to obtain service quality. After the user selection process, it is highly necessary to schedule the users with less wastage of bandwidth. To fulfil the demands of various multimedia traffic users and provide them application specific quality, the proposed TLUS scheme performs scheduling in two levels. At the first level, the active set of users is selected based on effective channel gains. At the second level, the users with close transmission durations are identified and the transmission of data will be done concurrently.

The brief description of the proposed scheme is as follows:

1) User selection at the first level:

The main objective of the user selection at the first level scheduling is to select the best users during CSI acquisition stage and reduce the overhead by limiting the contenting users.

For particular user $k$, determine $v_{k}$ the element of $h_{k}$ orthogonal to the subspace sized by $\left\{v_{(1)}, v_{(2)}, \ldots . ., v_{(|S|)}\right\}$

$$
v_{k}=h_{k}-\sum_{j=1}^{|S|} \frac{h_{k} v_{(j)}^{*}}{\left\|v_{(j)}\right\|^{2}} v_{(j)}
$$

The actual (or) effective channel gain of user $\mathrm{k}$ is expressed as $\mathrm{v}_{\mathrm{k}}$, it is seen that $\left\{v_{(j)}, 1 \leq j \leq|S|\right\}$ is a set of orthogonal vectors in $C^{1 \times M}$. ' $\mathrm{S}$ ' is the group of intended users. The primary projected element is usually selected as the best user in this algorithm.

Each user station calculates the alignment metric between channel state vector $h_{k}$ and the $i^{\text {th }}$ probe vector $p_{i}$

$$
g_{k}=\frac{\left\|h_{k} p_{i}\right\|^{2}}{\left\|h_{k}\right\|^{2}}
$$

The orthogonality requirement for $\mathrm{p}_{\mathrm{i}}$ is given by,

$$
\left.\begin{array}{l}
h_{k} \cdot p_{i}=0, k \in S \\
P=\operatorname{Null}\left(H_{n-1}\right)
\end{array}\right\}
$$

Where ' $\mathrm{P}$ ' is termed as probe vector and ' $\mathrm{n}$ ' is the series of contending rounds.

2) User grouping and scheduling at the second level:

During this level, the AP prepares the transmission schedule according to the transmission times required to serve the user traffic. By this process, the time spent on each individual user can be used efficiently. The two-level user scheduling process for the downlink multi-user scenario is depicted in Fig. 2. The AP first sends the NDPA and NDP frames to the user stations in order to initiate the channel sounding process. Then, the user stations respond with the corresponding channel information. The AP selects the users using channel state orthogonality and prepares the transmission schedule based on transmission durations. So, the selected users will be assigned to groups based on the similarity in download intervals for the requested application. The concurrent transmission enables users to receive data with less waiting time.

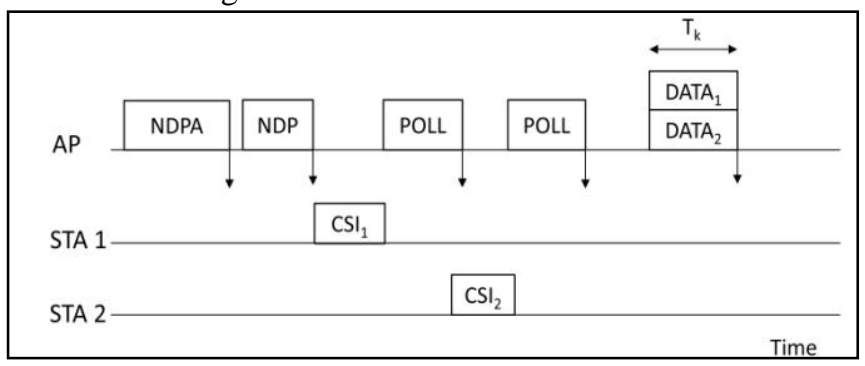

Fig. 2.DL-MU-MIMO Scheduling

The transmission duration $\left(\mathrm{T}_{\mathrm{k}}\right)$ of each user can be determined using the queue length information at the $\mathrm{AP}\left(\mathrm{Q}_{\mathrm{k}}\right)$ and the possible rate of transmission $\left(\mathrm{R}_{\mathrm{k}}\right)$. It is given by,

$$
T_{k}=\frac{Q_{k}}{R_{k}}
$$

Where, $\mathrm{k}=1,2,3, \ldots \ldots \ldots . \mathrm{N}$ be the number of contending user stations. The user time constraints varies in accordance with the required data demands. For example, a video user always require more transmission time since the number of packets will be huge. The best effort users generally require less amount of time to download the data. Here, in this algorithm the users with closest transmission time will be grouped and scheduled concurrently to utilize the resources efficiently. Some of the key considerations in the proposed system are given below:

- To avoid interference among the contending users, MMSE precoding is used in the algorithm.

- To show the robustness, frequency selective fading is assumed.

- To improve the overall throughput performance, 256-QAM and up to 8 access point antennas are considered in our system.

To understand the downlink transmission procedure, the step-wise description of TLUS scheme is given below. For the purpose of execution, an AP with $\mathrm{M}$ antennas, $\mathrm{K}$ user stations with one or more antennas and zero-mean unit-variance Gaussian channel is assumed. The AP computes the precoding matrix for the set of users using Eq. 2.

1. At the beginning, the AP initiates the polling process and user 1 responds with its CSI.

2. AP prepares the channel trace information and transmits it along with polling data.

3. Now, the remaining users participate in polling based on their individual channel gains and the next best user will be selected in this round.

4. The user stations will be selected based on satisfying the criteria from Eq. 5.

5. Repeat the steps from (2-4), till the end of polling process.

6. Now, the AP group the selected user stations based on the individual transmission durations computed from Eq. 6.

7. The AP places the data for transmission to the users. 
8. The user stations acknowledge the AP, after receiving the requested data.

\section{TLUS Algorithm}

In order to obtain an optimal user combination that maximizes the overall network throughput and provide fair allocation of resources, here TLUS employs a two level user scheduling process. For the given set of user receiver stations $\left\{\mathrm{k}_{1}, \mathrm{k}_{2}, \ldots \ldots \ldots \mathrm{k}_{\mathrm{N}}\right\}$ proposed scheme selects the best possible user group for the AP to simultaneous downlink data transmissions.

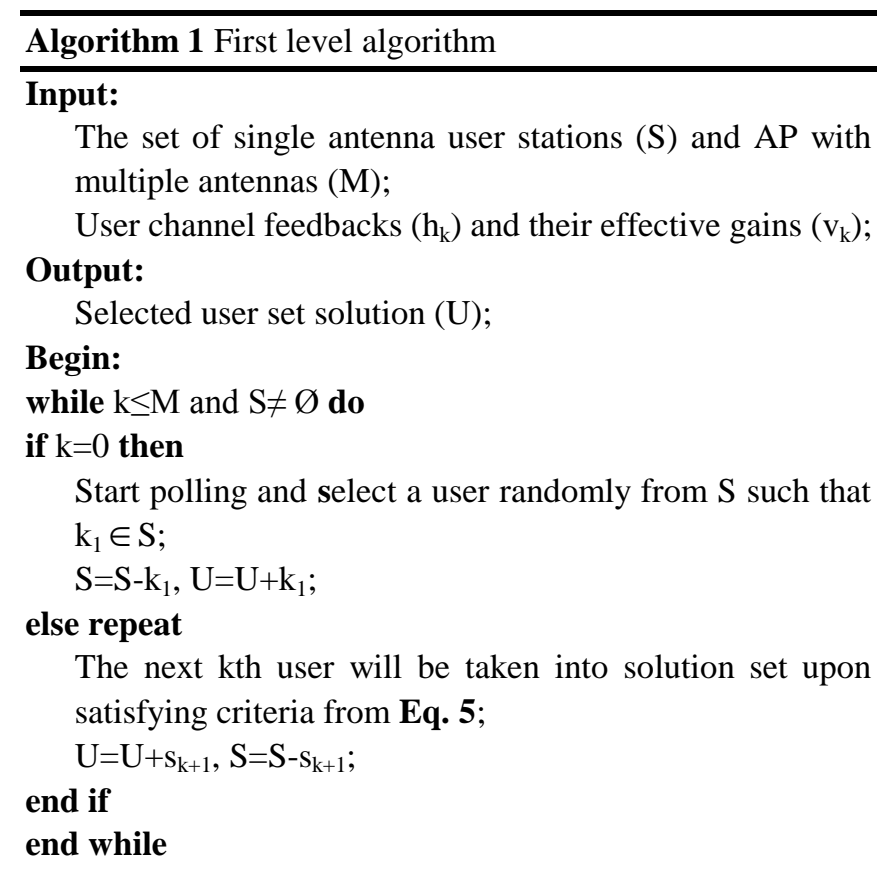

The Algorithm 1 is about the user selection process of TLUS. The input is the set of user stations with corresponding channel states. The output is the selected user solution set.

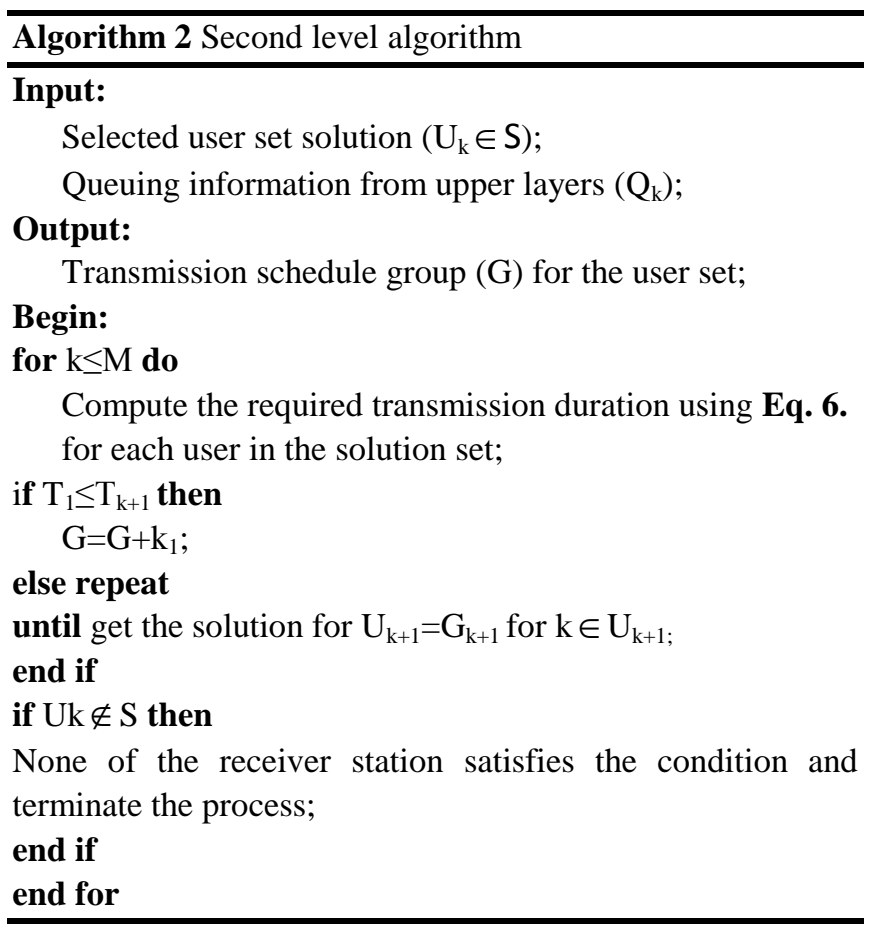

The Algorithm 2 is about the preparation of transmission schedule for preferable set of users with constraints on time durations. Cross-layer information is used for achieving better results.

\section{RESULTS AND DISCUSSION}

In this part, we evaluate the performance of the intended two-level user-scheduling scheme by comparing with three well-known algorithms JUSS[30], 802.11ac+[22] and RUS[19]. The simulation part is carried out using Matlab. The various parameters used for the simulation are given in Table. I. The evaluation is done for $M=4,8$ antennas at the access point. The number of users that can be served is ranging from 10 to 80 . The specified physical layer operating frequency is $5 \mathrm{GHz}$. The number of subcarriers is 108 and transmission power spans from 23 to $25 \mathrm{dBm}$. The channel aggregation bandwidth is chosen as $40 \mathrm{MHz}$. The data is generated in packets of size ranging from 512-1518 bytes. Here, we study the impact of number of users, use of multi-antennas, on the proposed QoS scheduling technique.

Table- I: Simulation parameters

\begin{tabular}{|l|l|}
\hline \multicolumn{1}{|c|}{ Parameters } & \multicolumn{1}{c|}{ Values } \\
\hline Carrier frequency & $5 \mathrm{GHz}$ \\
\hline RF Bandwidth & $40 \mathrm{MHz}$ \\
\hline Number of subcarriers & 108 \\
\hline Modulation scheme & $256-\mathrm{QAM}$ \\
\hline Payload length & $512-1518$ bytes \\
\hline Tx power & $23 \mathrm{dBm}$ \\
\hline MAC protocol & Extended CSMA/CA \\
\hline
\end{tabular}

\section{A. Throughput performance}

Here, we evaluate the effect of number of users on the throughput and demonstrate its performance over chosen techniques. Fig. 3 shows the throughput modelling of the proposed scheduling scheme for $\mathrm{M}=8$ antennas at the AP. It is clear from the result that, TLUS exhibits consistent throughput levels even when the network size scales up. It performs well when compared to JUSS and 802.11ac+. The mechanism of obtaining CSI, selecting the desired user stations, and allowing them to transmit in a scheduled manner has led to the improved throughput performance of the system. As RUS doesn't consider channel characteristics, packet queue information, and transmission duration, its throughput is low.

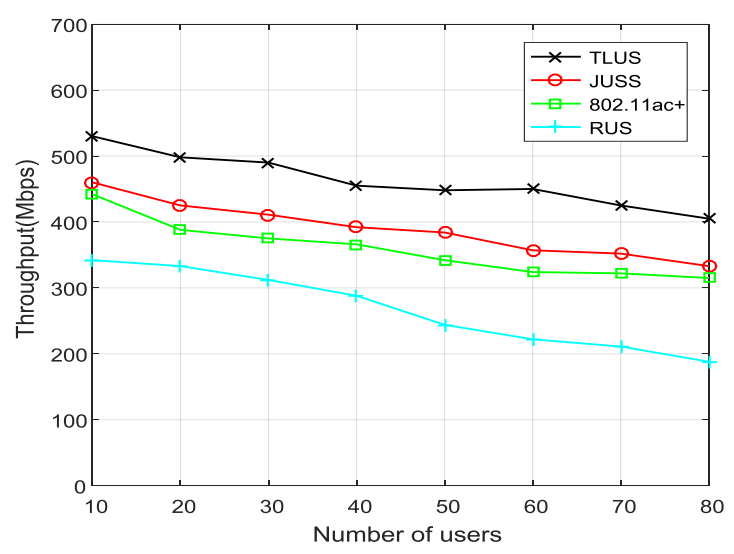

Fig. 3.Throughput analysis w.r.t number of users 


\section{QoS-Based Two-Level user Scheduling Scheme for MU-MIMO Wireless LANs}

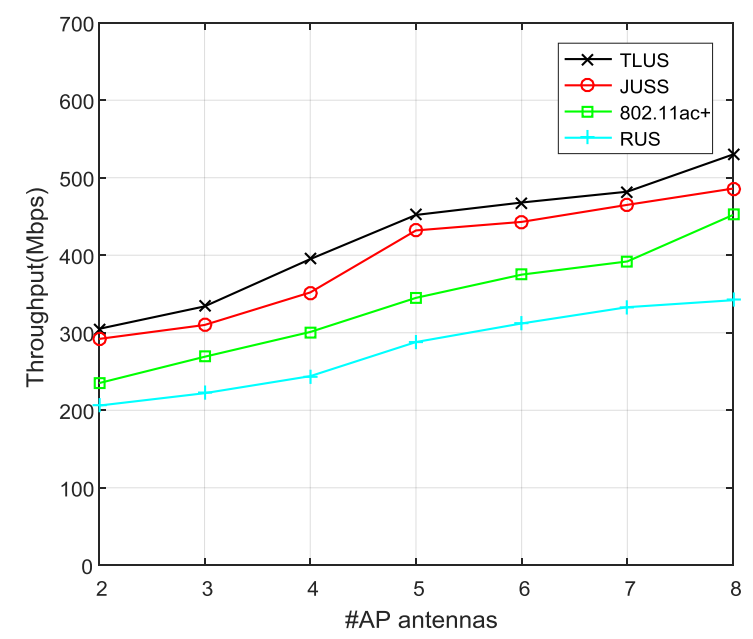

Fig. 4.Throughput analysis w.r.t number of AP antennas

The effect of the multiple antennas on the downlink throughput for each of the user-scheduling scheme is shown in Fig. 4. The number of transmitting antennas at the AP is varied from 2 to 8 , and the user stations is set to 10 . It is visible from the result that, the TLUS mechanism carry out better performance in contrast with other schemes. As the amount of transmitting antennas at AP increases, the throughput of various schemes can be greatly enhanced. Except RUS, the performance of all the considered protocols is similar as they collect the CSI from only intended users. This in turn reduces the CSI overhead and results in better performance.

\section{B. Impact of AP antennas on packet loss}

The use of multiple antennas at the access point may provide improved diversity. But, this will lead to increase in CSI overhead. Also, the number of bits needed to express the CSI is made large. Hence, leads to unwanted collisions and packet loss. The result obtained in Fig. 5. shows that there is a trade-off between packet loss and throughput. Although, there is an improvement in throughput due to spatial diversity and higher order modulation, packet loss cannot be ignored. So, along with high data rates, there may be loss or damage of packets due to collisions. Due to the random selection of users, RUS incurs more packet loss.

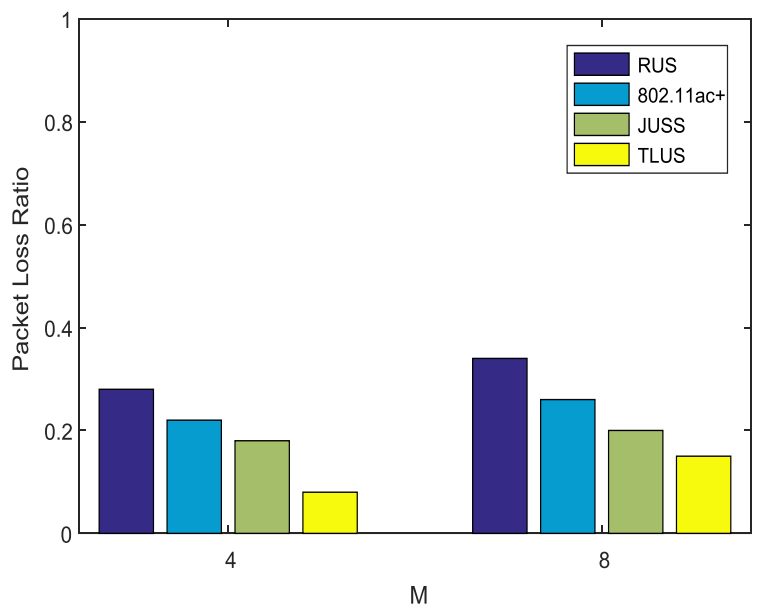

Fig. 5.Impact of antennas on packet loss

\section{Delay and fairness}

The fairness performance measure for the chosen protocols of the study is illustrated in Fig. 6. It can be viewed from result that, the MU-MIMO transmission performs well because it can able to assist several users simultaneously. In particular, TLUS with $\mathrm{M}=8$ attains close to precise fairness amongst users. On the whole, RUS and 802.11ac+ seems to provide low fairness when compared to TLUS scheduling protocol. TLUS scheme achieves fairness mainly due to the constraints adopted in user selection process and scheduling. The download transmission intervals depend on the type of multimedia requests. The way they are grouped and scheduled decides the final quality perceived.

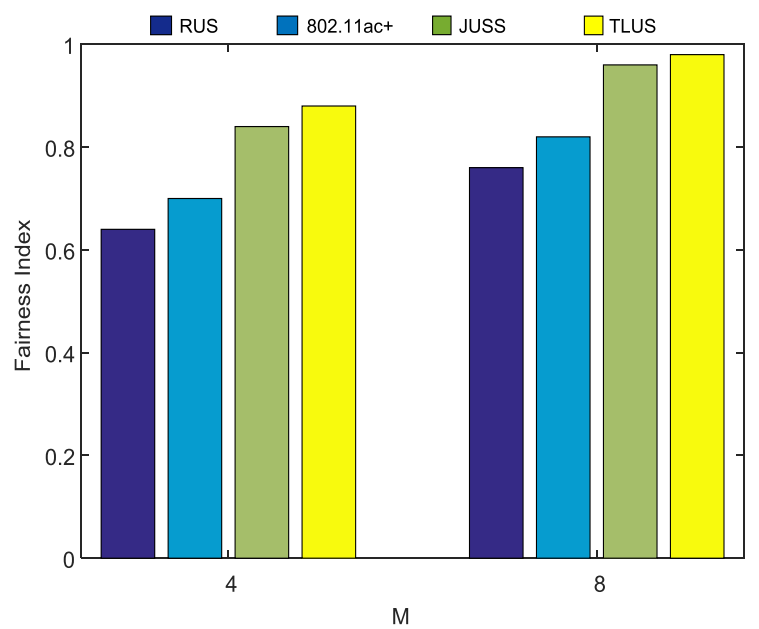

Fig. 6.Fairness Index

Next, we show the fairness performance comparison of all the four schemes as a function of number of users. As depicted in the Fig. 7. all the approaches achieve fairness close to 0.9 , when the user size is small. TLUS algorithm provides fairness, even when the network size scales up. This is possible due to the considerstion of other parameters in scheduling decision. While, for the remaining schemes, the fairness drops automatically with the increase in number of users. Overall, the proposed scheme provides optimal fairness over the chosen algoithms.

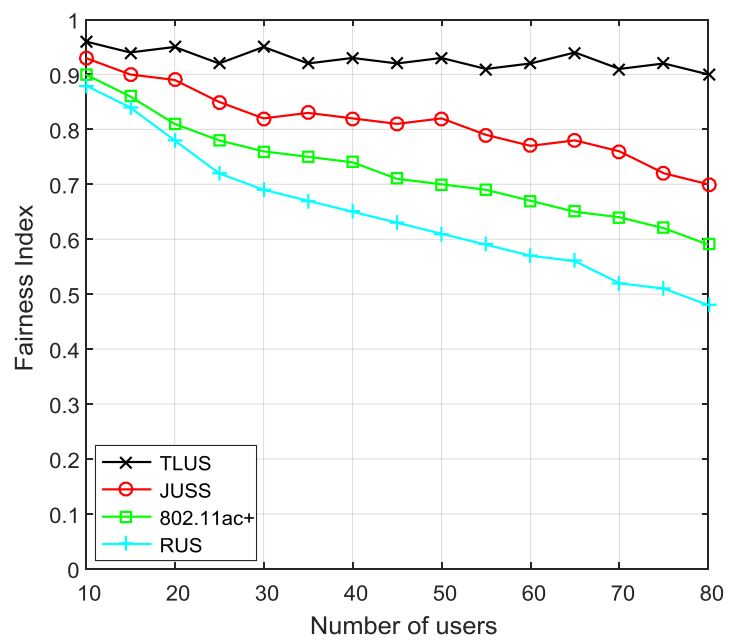

Fig. 7.Fairness w.r.t number of users 


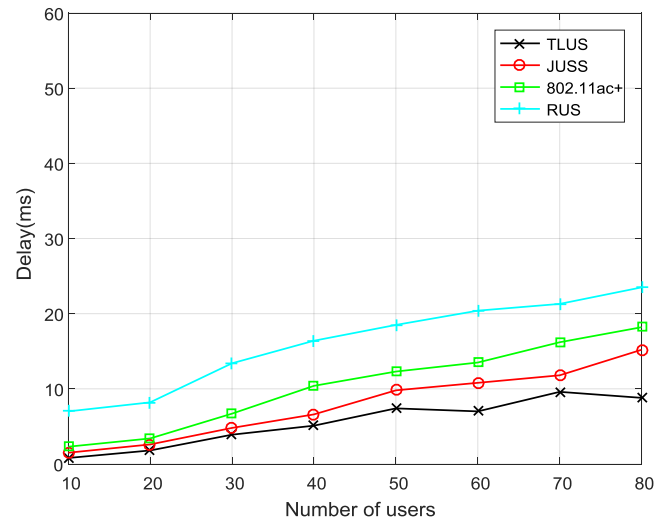

Fig. 8.Delay

Fig. 8 gives the packet delay curve with regard to the number of users. It is found clearly that, the overall delay in packet arrival is less for TLUS in comparison with RUS and $802.11 \mathrm{ac}+$. Because of the random and predefine selection of users, and lack of proper contention mechanism, 802.11ac protocol is not able to provide fairness and results in large delay. The interesting aspect here is, the delay may be more in some cases where the chances of transmitting in parallel are less. It is clear from the delay plot, that the delay in packet transmission looks high in TLUS at certain instants.

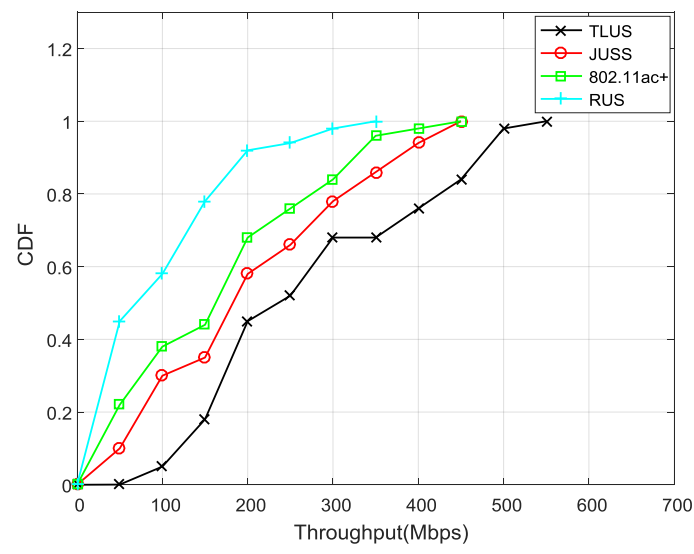

Fig. 9.Throughput fairness

To evaluate the throughput fairness among the users, (cummulative distribution function) CDF plot is obtained and corresponding distribution curves are illustrated in the Fig. 9. TLUS scheme achieves throughput gain over the other mechanisms. This is mainly due to the channel contention technique adopted, and the utilization of transmission time duration in the scheduling process. RUS scheme gives least performance, since the user selection is random and it doesn't consider channel characteristics and other constraints. Also, JUSS and 802.11ac+ exhibits almost similar performance. Because these schemes select and schedule the users just depending on the contention based feedback mechanisms. Some key inferences about the results are as follows:

- Throughput comparison shows that the proposed TLUS yields better result and it nearly achieves $2 x$ throughput gain over the other three scheduling schemes.

- With the rise in the quantity of users and transmit antennas, the proposed scheme achieves fairness above 0.9 .

- In addition, it also performs well against the other three schemes in QoS metrics like delay and packet loss.

\section{CONCLUSION}

In this paper, we have presented TLUS, a QoS based two-level user scheduling mechanism for the downlink data transmission of MU-MIMO WLANs. The user scheduling will be carried-out in two-levels. In the first level, the desired will be selected based on contention mechanism. In the second level, the transmission schedule is prepared by the AP based on time required for data transfer. Here, we investigate the throughput and fairness performance of 802.11 wireless LAN by studying the combined effect of scheduling with several physical layer aspects like channel information, modulation rate, and multiple antennas. For the purpose of comparison, some well-known scheduling algorithms are considered. TLUS achieves significant throughput gain and near to optimal fairness over other schemes. We also studied QoS aspects like impact of AP antennas on packet loss and delay. From the results, it is shown that, with controlled channel access and by employing advanced data rate enhancement techniques, the aggregate performance of the system can be greatly improved.

\section{REFERENCES}

1. Jim Geier, Designing and deploying 802.11 Wireless Networks, Cisco press, 2010.

2. A.J.Paulraj, D.A.Gore, R.U.Nabar,H.Bolcskei, "An overview of MIMO communications - a key to gigabit wireless," Proceedings of the IEEE vol.92, no.2, 2004.

3. Edward Au, "Exciting Projects for PHY and MAC Layers of IEEE 802.11," IEEE Vehicular technology magazine, vol.11, no.2, 2016.

4. D.Nojima, L.Lanante, Y.Nagao, M.Kurosaki, H.Ochi, "Performance evaluation for multi-user MIMO IEEE 802.11 ac wireless LAN system," 14th International Conference on Advanced Communication Technology (ICACT), Pyeong Chang, South Korea, 2012.

5. R.Pierre F.Hoefel, "Multi-user OFDM MIMO in IEEE 802.11ac WLAN: A simulation framework to analysis and synthesis", IEEE Latin-America Conference on Communications, Santiago, Chile, 2013.

6. G.Z.Khan, R.Gonzalez, E.C.Park, "A performance analysis of MAC and PHY layers in IEEE 802.11ac wireless network," 18th International Conference on Advanced Communication Technology (ICACT), Pyeongchang, South Korea, 2016.

7. B.Bellalta, J.Barcelo, D.Staehle, A.Vinel, M.Oliver, "On the performance of packet aggregation in IEEE 802.11 ac MU-MIMO WLANs", IEEE communications letters, vol. 16, no. 10, october 2012.

8. J.Cha, H.Jin, B.Chul Jung, D.K.Sung, "Performance comparison of downlink user multiplexing schemes in IEEE 802.11 ac: Multi-user MIMO vs. frame aggregation”, IEEE Wireless Communications and Networking Conference (WCNC), Shanghai, China, 2012.

9. Y.Nomura, K.Mori, H.Kobayashi, "Efficient frame aggregation with frame size adaptation for next generation MU-MIMO WLANs", 9th International Conference on Next Generation Mobile Applications, Services and Technologies, Cambridge, UK, 2015.

10. M.X.Gong, E.Perahia, R.Want, S.Mao, "Training protocols for multi-user MIMO wireless LANs", 21st Annual IEEE International Symposium on Personal, Indoor and Mobile Radio Communications, Instanbul, Turkey, 2010.

11. X.Xie, X.Zhang, K.Sundaresan, "Adaptive feedback compression for MIMO networks", Proceedings of the 19th annual international conference on Mobile computing \& networking, Miami, Florida, USA, 2013.

12. H. Lou, M. Ghosh, P. Xia, and R. Olesen, "A comparison of implicit and explicit channel feedback methods for MU-MIMO WLAN systems", 24th Annual International Symposium on Personal, Indoor, and Mobile Radio Communications (PIMRC), pp. 419-424, 2013.






\section{QoS-Based Two-Level user Scheduling Scheme for MU-MIMO Wireless LANs}

13. C.Zhu, A.Bhatt, Y.Kim, O.A.Magd, C.Ngo,"MAC enhancements for downlink multi-user MIMO transmission in next generation WLAN", IEEE Consumer Communications and Networking Conference (CCNC), Las Vegas, NV, USA, 2012.

14. M.Yazid, A.Ksentini, L.B.Medjkoune, D.Aïssani,"Performance analysis of the TXOP sharing mechanism in the VHT IEEE 802.11 ac WLANs", IEEE Communications Letters, vol.18, no.9, 2014.

15. M.Aajami, J.B.Suk, "Optimal TXOP Sharing in IEEE 802.11ac", IEEE Communications Letters, vol.19, no.7, 2015.

16. S. Mangold, S. Choi, G. R. Hiertz, O. Klein, and B. Walke, "Analysis of IEEE 802.11e for QoS support in wireless LANs," Wireless Communications, IEEE, vol. 10, no. 6, pp. 40-50, 2003.

17. G. Redieteab, L. Cariou, P. Christin, and J. F. Helard, "PHY+MAC channel sounding interval analysis for IEEE 802.11ac MUMIMO", in Proc. 9th IEEE Int. Symp. on Wireless Commun. Syst. ISWCS 2012 , Paris, France, 2012.

18. O. Bejarano, E. Magistretti, O. Gurewitz, and E. W. Knightly, "MUTE: sounding inhibition for MU-MIMO WLANs", in Proc. 11th Ann. IEEE Int. Conf. on Sensing, Commun., and Network. SECON, Singapore, Singapore, 2014, pp. 135-143.

19. IEEE, IEEE Std P802.11ac: Part 11: Wireless LAN Medium Access Control (MAC) and Physical Layer (PHY) specifications: enhancements for very high throughput for operation in bands below $6 \mathrm{GHz}, 2013$.

20. S. Huang, H. Yin, J. Wu, V. Leung, "User selection for multiuser MIMO downlink with zero-forcing beamforming," IEEE Transactions on Vehicular Technology, vol. 62, no. 7, pp. 3084-3097, 2013

21. X. Xie and X. Zhang, "Scalable user selection for MU-MIMO networks," in Proc. of IEEE International Conference on Computer Communications (INFOCOM), pp. 808-816, 2014.

22. K. Lee and C. Kim, "User scheduling for MUMIMO transmission with active CSI feedback", EURASIP journal on Wireless Communication and Networking, vol. 112, 2015.

23. A. Zhou, T. Wei, X. Zhang and et al., "Signpost: Scalable MU-MIMO Signaling with Zero CSI Feedback," in Proc. of ACM International Symposium on Mobile Ad Hoc Networking and Computing (MobiHoc), pp. 327-336, 2015.

24. Y. Zhou, A. Zhou, and M. Liu, "OUS: Optimal user selection in MU-MIMO WLANs", in Proc. Int. Conf. on Computer Network. and Comm. ICNC 2016, Kauai, HI, USA, 2016.

25. N.Anand, J.Lee, S.J.Lee, E.W.Knightly, "Mode and User Selection for Multi-User MIMO WLANs without CSI", IEEE Conference on Computer Communications (INFOCOM), 2015.

26. M.Esslaoui, F.Riera-Palou and G.Femenias, "A fair MU-MIMO scheme for IEEE 802.11ac", International Symposium on Wireless Communication Systems (ISWCS), 2012.

27. Kyeongjun Ko and Jungwoo Lee, "Multiuser MIMO User Selection Based on Chordal Distance", IEEE transactions on communications, Vol. 60, No. 3, March 2012.

28. Shanshan Wu, Wenguang Mao, and Xudong Wang, "Performance Study on a CSMA/CA-Based MAC Protocol for Multi-User MIMO Wireless LANs", IEEE transactions on wireless communications, vol. 13, no. 6, June 2014.

29. Suhua Tang, "Distributed Multiuser Scheduling for Improving Throughput of Wireless LAN", IEEE transactions on wireless communications, vol. 13, no. 5, May 2014.

30. D.Srinivasa Rao, V.Berlin Hency, "QoS-based joint user selection and scheduling for MU-MIMO WLANs", Journal of telecommunication and information technology, vol.4, 2017.

\section{AUTHORS PROFILE}

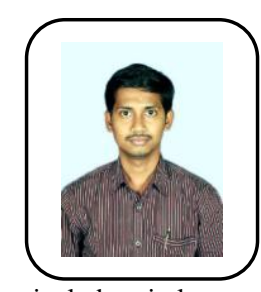

D. Srinivasa Rao has received his Master's degree in communication systems from Thiagarajar College of Engineering, Madurai. Now, he is pursuing Ph.D. from Vellore Institute of Technology, Chennai. He has nearly ten years of experience in teaching. He is currently working as an Asst. Professor in Dept. of ECE, GMR Institute of Technology, Rajam, India. His research interests include wireless communications, next generation networks, and coding techniques.

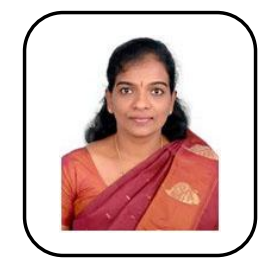

V. Berlin Hency has received BE degree in Electronics and Communication Engineering from Manonmaniam Sundaranar University. She has received Master's degree in Applied electronics from Sathyabama University. She has received $\mathrm{PhD}$ degree from Anna University in Information and Communication Engineering. She is an Associate Professor in the School of Electronics Engineering, Vellore Institute of Technology, Chennai. Her research interests include Wireless Networks and IoT. 